\title{
28 Research Square \\ NCC-BCBM, a Nomogram Prognostic Model in Breast Cancer Patients with Bone Metastases
}

Jie Zhai

Chinese Academy of Medical Sciences Cancer Institute and Hospital: Cancer Hospital Chinese Academy of Medical Sciences

Qiang Liu

Chinese Academy of Medical Sciences Cancer Institute and Hospital: Cancer Hospital Chinese Academy of Medical Sciences

\section{Ping Bai}

Chinese Academy of Medical Sciences Cancer Institute and Hospital: Cancer Hospital Chinese Academy of Medical Sciences

\section{Zhongzhao Wang}

Chinese Academy of Medical Sciences Cancer Institute and Hospital: Cancer Hospital Chinese Academy of Medical Sciences

\section{Yi Fang}

Chinese Academy of Medical Sciences Cancer Institute and Hospital: Cancer Hospital Chinese Academy of Medical Sciences

Jing Wang ( $\nabla$ wangjing@cicams.ac.cn )

Cancer Institute and Hospital, Chinese Academy of Medical Sciences and Peking Union Medical College https://orcid.org/0000-0002-3224-3993

\section{Research article}

Keywords: Bone metastasis, Prognosis, Predictors, Nomogram, Breast cancer

Posted Date: April 12th, 2021

DOI: https://doi.org/10.21203/rs.3.rs-384643/v1

License: (c) (1) This work is licensed under a Creative Commons Attribution 4.0 International License. Read Full License 


\section{Abstract}

Purpose Accurate prediction tools to facilitate risk stratification and therapeutic strategies for breast cancer patients with bone metastasis (BCBM) are lacking. We constructed and validated a new nomogram prognostic model, named NCC-BCBM, for breast cancer patients with bone metastasis using a large BCBM cohort from the SEER (Surveillance Epidemiology and End Results) database.

Patients and Methods Clinical information for 8655 patients diagnosed from 2011 to 2013 was collected to develop the nomogram prognostic model. The predictive accuracy and discriminative ability of the nomogram were evaluated by concordance index (C-index) and calibration curve. The model was further validated in an independent cohort of 4634 BCBM patients diagnosed from 2014 to 2015.

Results The following clinical variables were enrolled in the final prognostic model: age, race, surgery, radiation therapy, chemotherapy, laterality, grade, molecular subtype, American Joint Committee on Cancer (AJCC T) stage, AJCC N stage and extra metastatic sites except bone. The C-index for the developed model in training cohort was $0.702(95 \% \mathrm{Cl}, 0.696$ to 0.709$)$. The calibration curve for probability of 1-year, 3-year and 5-year survival showed good agreement between prediction by nomogram and direct observation. The prognostic model was validated in an independent validation cohort with a concordance index of $0.748(95 \% \mathrm{Cl}, 0.737$ to 0.759$)$.

Conclusion We developed and validated a nomogram prognostic model for BCBM patients and the proposed nomogram resulted in good performance.

\section{Introduction}

Breast cancer (BC) is the most common cancer and reached the first leading cause of cancer-related mortality among females worldwide.[1] The most common metastasis of $\mathrm{BC}$ is bone metastasis, and $65 \%$ to $75 \%$ of patients with metastatic BC suffer bone metastasis, which has a directly negative effect on patients survival, a marked increase in the mortality rate.[2-5] Bone metastases increase mortality and decrease health-related quality of life.[6] Therefore, early prognostic assessment, which could be a key evidence of clinical monitor, is important. Sufficient studies have reported the incidence and survival of bone metastasis in breast cancer. A study with a large cohort of 7064 breast cancer patients showed that $589(22 \%)$ of them developed bone metastases during a mean of 8.4 years and the median survival after diagnosis of bone metastasis was 2.3 years in women with bone-only metastases, while in women with visceral and bone metastases the median survival was $<1$ year.[7] Another study showed median overall survival (OS) from bone metastasis diagnosis was 40 months in BC patients. [8]. Several studies previously summarized risk factors and predictive factors from clinical data by creating prediction models. In patients with stage II and III breast cancer, results showed positive predictive values for bone metastases were 92.3\% (95\% Cl 69.3-99.2\%), but had low sensitivity. Age, stage, hormone receptor status, and cancer treatment were included in the predictors.[9] A small cohort containing 169 cases were used to construct a model to predict patients' risk of developing bone metastases. The variables in this 
model consisted of 8 proteins and lymph node status.[10] MD Anderson cancer center constructed a clinical nomogram to predict subsequent bone-only metastasis with a 4175-patient-training cohort.[11] This nomogram suggested that age, lymph node status, lymphovascular space invasion, T classification, and hormone receptor status were independently associated with subsequent bone metastasis in breast cancer patients. However, an external study that aimed to validate the MDACC nomogram showed limitations in its generalizability to a new and independent European patient group. [12] There are also studies created models using genomic copy number and transcriptomes to predict bone metastasis in earlystage breast cancer.[13-15]. These studies have small cohort and it is difficult to apply them directly into daily clinical practice. More importantly, these studies are all focused on predicting the subsequent bone metastasis in $\mathrm{BC}$ patients.

Large population-based prognosis assessment tool for BCBM is lacking. The characteristics of patients, as well as the prognosis factors among patients have increased the difficulties in assessing the prognosis and devising treatment plans. Thus, Further study is needed. Therefore, the population-based prediction of prognosis for newly diagnosed breast cancer bone metastasis (BCBM) is required for helping clinicians make decisions and optimize therapeutic options.

Herein, we tried to focused on the individual prognosis of patients diagnosed with bone metastasis. In the present study, we developed and validated a nomogram prognostic model in a population of 13289 patients. We investigated the sociodemographic and clinicopathologic predictors associated with BCBM, and developed a robust nomogram model for predicting BCBM survival at 1 year, 3 years and 5 years. The proposed prognostic model was validated in an independent external validation cohort and showed good performance.

\section{Methods}

\section{Study population and design}

Since clinical records on the molecular subtypes and sites of distant metastasis collected in SEER database was started in 2010, only BCBM diagnosed from 2010 to 2015 were enrolled in the present study. Inclusion criteria were set as follows: 1) patients with bone metastasis; 2) follow-up information is clear; 3) reporting source was neither autopsy nor death certificate only. Exclusion criteria were set as follows: 1) cases with tumors of uncertain origin; 2) patients with duplicated record. A total of 8655 cases diagnosed from 2010 to 2013 were allocated to the training cohort and were used to build the nomogram prognostic model. Another independent 4634 cases diagnosed from 2014 to 2015 were allocated to the independent validation cohort and used to verify the effectiveness of the model.

\section{Variable Selection}

The primary outcome was overall survival which was defined as the length of time from diagnosis to death or last follow-up. The following variable data were extracted and classified according to the codes in the SEER database: sex, age, race, marital status at diagnosis, insurance recode[16], breast tumor 
laterality, tumor primary site, molecular subtype, histological grade, pathological pattern including infiltrating duct carcinoma (IDC), lobular carcinoma (LC), infiltrating ductal and lobular carcinoma (IDLC), cribriform carcinoma, tubular adenocarcinoma, mucinous adenocarcinoma, infiltrating duct mixed with other types of carcinoma (IDM), ductal carcinoma, micropapillary, and other, American Joint Committee on Cancer (AJCC) T stage, AJCC N stage, surgery recode, radiation recode, chemotherapy recode, and survival in months, and number of extracranial metastatic sites.

\section{Statistical Analysis}

The nomogram was developed based on the results of multivariate Cox regression analyses via the rms package [17] of in R version 3.6.3 (http://www.r-project.org/ ). The final model selection was performed by a backward stepdown selection process by the Akaike information criterion [18]. The effectiveness of the nomogram was determined by concordance index (C-index) and assessed by comparison against observed Kaplan-Meier estimates of survival probability. Bootstraps with 1,000 resample were used for these activities. C-index and 95\% Confidence Interval $(\mathrm{Cl})$ were calculated using survcomp package [19] in $\mathrm{R}$. The calibration plots were generated by comparing the nomogram-predicted probability of overall survival at 1 year, 3 years and 5 years with the observed survival probability. Interpretation of C-index has similar characteristics to a receiver-operator curve: a value of 1.0 suggests a model that is perfectly concordant with the dataset; a value of 0.0 indicates perfect discordance [20]. $\mathrm{P}<0.05$ was considered statistically significant.

\section{Results}

\section{Characteristics of the Training and Validation Cohorts}

A total of 13289 cases that exclued those having any missing variables were included in this study. Included cases were divided into two distinct groups based on year of diagnosis: cases diagnosed from 2010 to 2013 ( $n=8635$ ) were used as the training cohort, whereas cases diagnosed from 2014 to 2015 $(n=4634)$ were used as the validation cohort. The median follow-up time was 4.75 years $(95 \% \mathrm{Cl}$ : $4.67-$ 4.83 years) for the training cohort and 1.92 years (95Cl\%:1.83-1.92 years) for the testing cohort. Clinicopathological characteristics of the two datasets are summarized in Table 1.

\section{Nomogram Prognostic Model in Training Cohort}

Results of the univariate analysis are presented in Table S1. Multivariate Cox analyses revealed that age, race, surgery, radiation therapy, chemotherapy, laterality, grade, molecular subtype, AJCC T stage, AJCC N stage and extra metastatic sites except bone were independent risk factors for OS (Table 2). The prognostic nomogram model that included all significant independent factors for OS in the primary cohort is shown in Figure 1. The C-index for OS prediction was 0.702 (95\% Cl, 0.696 to 0.709). Additionally, the calibration plot for the probability of survival at 1 year, 3 years and 5 years showed a good agreement between the prediction by nomogram and actual observation (Fig $2 \mathrm{~A} ; \mathrm{C}$ and $\mathrm{E}$ ). 


\section{External Validation of the Nomogram}

In the validation cohort, we test the nomogram prognostic model using the same model parameters as the proposed nomogram model in the training cohort. Surprisingly, we found the C-index of the nomogram for predicting OS was $0.748(95 \% \mathrm{Cl}, 0.737$ to 0.759$)$, and the calibration curve also showed excellent agreement between prediction and observation in the survival probability of 1 year, 3 years and 5 years (Fig 2B; D and F). These results indicated that prediction performance in an independent data set were excellent and therefore confirmed the exportability of the model.

\section{Discussion}

In the present study, the NCC-BCBM prognostic model was constructed and validated by using a large cohort of BCBM cases across the United States. This NCC-BCBM nomogram, based on currently available demographic, staging, and clinical therapeutic information can predict the survival probability for individual BCBM, which might be helpful for assisting clinicians in making therapeutic strategies.

\section{Prognostic Predictors for BCLM}

Previous population-based studies summarized prognostic factors and survival outcomes of breast cancer liver metastasis (BCLM). [21-24] Results showed histological grade 3 disease at primary presentation, advanced age and ER negative tumors predicted poor prognosis. However, cohort of these studies were small. A population-based study with large cohort analyzed factors for predicting the prognosis for BCLM.[25] This study included 2098 cases and explored predictive factors with KaplanMeier analysis and multivariate analysis. Results showed age at diagnosis, marital status, surgery, tumor subtype, bone metastasis and brain metastasis were independent prognosis factors from a competing risk mode. Factors in this study including age, surgery, extra metastatic sites and tumor subtypes are consistent with the independent risk factors in our cohort. We also found race, radiation therapy, chemotherapy, laterality, grade AJCC T stage, and AJCC N stage were independent risk factors for OS. Generally, we found more independent risk factors for OS in our BCBM study than those of BCLM, and the reason might be the large cohort.

\section{Nomogram prognostic model for BCBM}

Several previous studies have created nomograms for patients with BCBM. Delpech et. al [11] retrospectively collected 314 BCBM patients and constructed the first clinical nomogram using Cox proportional hazards regression model to predict BCBM. Although the nomogram was validated and the concordance index was reported 0.69 in training group and 0.73 in external validation group, the cohort was too small to perform an accurate prediction. Another study analyzed BCBM patients from SEER database, created a predictive nomogram with 3311 cases from training cohort and validated the nomogram with 2549 cases from validation cohort. [26] Bootstraps with 1,000 resamples were also used to validate the nomograms in both cohorts. This study had the largest cohort with satisfied C-index of 0.705 and 0.678 in training and validation cohort, respectively, and could assist clinicians in predicting 
survival. Huang et.al[27] analyzed risk factors of $\mathrm{BC}$ patients who developed bone metastasis with newly diagnosed infiltrating duct carcinoma (IDC), created a prognostic nomogram and validated it by ROC analysis. The AUCs showed $0.775,0.758$, and 0.731 in the training cohort and $0.770,0.773$, and 0.753 in the internal validation cohort; and $0.756,0.764$, and 0.767 in the external validation cohort for $1-, 3-$, and 5 -year OS, respectively. Generally, studies above used small cohorts for both building and validating the nomograms. In our study, we used 8635 cases as the training cohort and 4634 cases as the independent cohort. To the best of our knowledge, our study has the largest cohort of creating the nomogram and the largest cohort of validating the nomogram. C-index is a natural extension of ROC curve area.[28] Our Cindex for OS prediction reached 0.748 , notably higher than previous studies.

\section{Potential limitations}

Unavoidably, several flaws and limitations should be acknowledged in present study. First, this study only included patients with complete information, thus, selection bias might exist. Second, the SEER database does not have specific records about systemic therapy like endocrine and targeted therapy. Third, present study is retrospective, and internal and external validations were performed within SEER database.

Therefore, prospective and external validations in cohorts outside SEER database are further needed.

\section{Conclusions}

The current study developed a robust model to predict survival of breast cancer patients with bone metastasis using Cox proportional hazards regression in conjunction with a nomogram representation. The NCC-BCBM model is built with a combination of 10 clinical and molecular features in patients with breast cancer. This nomogram should be readily available to clinicians; validation results of the present study showed that this model should be highly repeatable and reproducible in similar patient groups.

\section{Declarations}

\section{Ethics approval and consent to participate}

All procedures performed in studies involving human participants were in accordance with the ethical standards of the institutional and/or national research committee and with the 1964 Helsinki declaration and its later amendments or comparable ethical standards.

\section{Consent for publication}

Not applicable.

\section{Availability of supporting data}

The data generated or analyzed during this study are included in this article, or if absent are available from the corresponding author upon reasonable request. 


\section{Competing interests}

No potential conflicts of interest were disclosed.

\section{Funding}

This paper was partially sponsored by grants from the National Natural Science

Foundation of China, No. 81872160 (Jing Wang).

\section{Authors Contributions}

Conception and design: Jing Wang (JW), Yi Fang (YF), Zhongzhao Wang (ZW), Qiang Liu (QL). Development of methodology: Jie Zhai (JZ) and QL. Acquisition of data: Ping Bai (PB). Analysis and interpretation of data: JZ, PB and QL; Writing, review and/or revision of the manuscript: JZ, QL, PBC. Administrative, technical, or material support: JZ, PB, QL. Study supervision: JW, YF, ZW. All authors read and approved the final manuscript.

\section{Acknowledgements}

Not applicable.

\section{References}

1. Bray, F., et al., Global cancer statistics 2018: GLOBOCAN estimates of incidence and mortality worldwide for 36 cancers in 185 countries. CA Cancer J Clin, 2018. 68(6): p. 394-424.

2. Coleman, R.E., Metastatic bone disease: clinical features, pathophysiology and treatment strategies. Cancer Treat Rev, 2001. 27(3): p. 165-76.

3. Mackiewicz-Wysocka, M., M. Pankowska, and P.J. Wysocki, Progress in the treatment of bone metastases in cancer patients. Expert Opin Investig Drugs, 2012. 21(6): p. 785-95.

4. Coleman, R.E., P. Smith, and R.D. Rubens, Clinical course and prognostic factors following bone recurrence from breast cancer. Br J Cancer, 1998. 77(2): p. 336-40.

5. Savci-Heijink, C.D., et al., Retrospective analysis of metastatic behaviour of breast cancer subtypes. Breast Cancer Res Treat, 2015. 150(3): p. 547-57.

6. Yang, M., C. Liu, and X. Yu, Skeletal-related adverse events during bone metastasis of breast cancer: current status. Discov Med, 2019. 27(149): p. 211-220.

7. Harries, M., et al., Incidence of bone metastases and survival after a diagnosis of bone metastases in breast cancer patients. Cancer Epidemiol, 2014. 38(4): p. 427-34.

8. Kuchuk, I., et al., Incidence, consequences and treatment of bone metastases in breast cancer patients-Experience from a single cancer centre. J Bone Oncol, 2013. 2(4): p. 137-44. 
9. Cronin-Fenton, D., et al., Breast cancer recurrence, bone metastases, and visceral metastases in women with stage II and III breast cancer in Denmark. Breast Cancer Res Treat, 2018. 167(2): p. 517528.

10. Hayashi, N., et al., Reverse-phase protein array for prediction of patients at low risk of developing bone metastasis from breast cancer. Oncologist, 2014. 19(9): p. 909-14.

11. Delpech, Y., et al., Clinical nomogram to predict bone-only metastasis in patients with early breast carcinoma. Br J Cancer, 2015. 113(7): p. 1003-9.

12. Ouldamer, L., et al., Intrinsic and extrinsic flaws of the nomogram predicting bone-only metastasis in women with early breast cancer: An external validation study. Eur J Cancer, 2016. 69: p. 102-109.

13. Liu, Y., et al., Genomic copy number imbalances associated with bone and non-bone metastasis of early-stage breast cancer. Breast Cancer Res Treat, 2014. 143(1): p. 189-201.

14. Neckmann, U., et al., GREM1 is associated with metastasis and predicts poor prognosis in ERnegative breast cancer patients. Cell Commun Signal, 2019. 17(1): p. 140.

15. Pavlovic, M., et al., Enhanced MAF Oncogene Expression and Breast Cancer Bone Metastasis. J Natl Cancer Inst, 2015. 107(12): p. djv256.

16. AA, A., et al., Cancer-specific outcomes among young adults without health insurance. Journal of clinical oncology : official journal of the American Society of Clinical Oncology, 2014. 32(19): p. 2025-30.

17. Harrell Jr, F.E., rms: Regression Modeling Strategies. R package version 4.0-0. City, 2013.

18. Sakamoto, Y., M. Ishiguro, and G. Kitagawa, Akaike information criterion statistics. Dordrecht, The Netherlands: D. Reidel, 1986. 81.

19. Schröder, M.S., et al., survcomp: an R/Bioconductor package for performance assessment and comparison of survival models. Bioinformatics, 2011. 27(22): p. 3206-3208.

20. Harrell Jr, F.E., K.L. Lee, and D.B. Mark, Multivariable prognostic models: issues in developing models, evaluating assumptions and adequacy, and measuring and reducing errors. Statistics in medicine, 1996. 15(4): p. 361-387.

21. O'Reilly, S.M., M.A. Richards, and R.D. Rubens, Liver metastases from breast cancer: the relationship between clinical, biochemical and pathological features and survival. Eur J Cancer, 1990. 26(5): p. 574-7.

22. Atalay, G., et al., Clinical outcome of breast cancer patients with liver metastases alone in the anthracycline-taxane era: a retrospective analysis of two prospective, randomised metastatic breast cancer trials. Eur J Cancer, 2003. 39(17): p. 2439-49.

23. Fendler, W.P., et al., Safety, Efficacy, and Prognostic Factors After Radioembolization of Hepatic Metastases from Breast Cancer: A Large Single-Center Experience in 81 Patients. J Nucl Med, 2016. 57(4): p. 517-23.

24. Wyld, L., et al., Prognostic factors for patients with hepatic metastases from breast cancer. $\mathrm{Br} \mathrm{J}$ Cancer, 2003. 89(2): p. 284-90. 
25. Chen, Q.F., et al., Prognostic factors and survival according to tumor subtype in newly diagnosed breast cancer with liver metastases: A competing risk analysis. Mol Clin Oncol, 2019. 11(3): p. 259269.

26. Wang, Z., et al., Novel prognostic nomograms for female patients with breast cancer and bone metastasis at presentation. Ann Transl Med, 2020. 8(5): p. 197.

27. Huang, Z., et al., Risk factors, prognostic factors, and nomograms for bone metastasis in patients with newly diagnosed infiltrating duct carcinoma of the breast: a population-based study. BMC Cancer, 2020. 20(1): p. 1145.

28. Pencina, M.J. and R.B. D'Agostino, Overall C as a measure of discrimination in survival analysis: model specific population value and confidence interval estimation. Stat Med, 2004. 23(13): p. 210923.

\section{Tables}


Table 1.

Demographics and Clinicopathologic Characteristics of Breast Cancer Patients With Bone Metastasis

\begin{tabular}{|c|c|c|c|}
\hline & Traning & Validation & Overall \\
\hline & $(n=8655)$ & $(n=4634)$ & $(n=13289)$ \\
\hline \multicolumn{4}{|l|}{ Age_group } \\
\hline$<40$ & $521(6.0 \%)$ & $293(6.3 \%)$ & $814(6.1 \%)$ \\
\hline $40-49$ & $1132(13.1 \%)$ & $517(11.2 \%)$ & $1649(12.4 \%)$ \\
\hline $50-59$ & $2107(24.3 \%)$ & $1143(24.7 \%)$ & $3250(24.5 \%)$ \\
\hline $60-69$ & $2299(26.6 \%)$ & $1250(27.0 \%)$ & $3549(26.7 \%)$ \\
\hline 70-79 & $1497(17.3 \%)$ & $868(18.7 \%)$ & $2365(17.8 \%)$ \\
\hline$>=80$ & $1099(12.7 \%)$ & $563(12.1 \%)$ & $1662(12.5 \%)$ \\
\hline \multicolumn{4}{|l|}{ Sex } \\
\hline Male & $100(1.2 \%)$ & $69(1.5 \%)$ & $169(1.3 \%)$ \\
\hline Female & $8555(98.8 \%)$ & $4565(98.5 \%)$ & $13120(98.7 \%)$ \\
\hline \multicolumn{4}{|l|}{ Race } \\
\hline White & $5868(67.8 \%)$ & 3057 (66.0\%) & $8925(67.2 \%)$ \\
\hline Black & $1299(15.0 \%)$ & 717 (15.5\%) & $2016(15.2 \%)$ \\
\hline Hispanic & $888(10.3 \%)$ & $492(10.6 \%)$ & $1380(10.4 \%)$ \\
\hline Asian/Pacific Islander & $534(6.2 \%)$ & $328(7.1 \%)$ & $862(6.5 \%)$ \\
\hline Other & $66(0.8 \%)$ & $40(0.9 \%)$ & $106(0.8 \%)$ \\
\hline \multicolumn{4}{|l|}{ Marital_status } \\
\hline None-single & $6344(73.3 \%)$ & 3319 (71.6\%) & $9663(72.7 \%)$ \\
\hline Single & $1837(21.2 \%)$ & $1073(23.2 \%)$ & $2910(21.9 \%)$ \\
\hline Unknown & $474(5.5 \%)$ & $242(5.2 \%)$ & $716(5.4 \%)$ \\
\hline \multicolumn{4}{|l|}{ Insurance } \\
\hline Uninsured & $418(4.8 \%)$ & $136(2.9 \%)$ & $554(4.2 \%)$ \\
\hline Insured & $8034(92.8 \%)$ & $4401(95.0 \%)$ & 12435 (93.6\%) \\
\hline Unknown & $203(2.3 \%)$ & 97 (2.1\%) & $300(2.3 \%)$ \\
\hline Laterality & & & \\
\hline
\end{tabular}




\begin{tabular}{|c|c|c|c|}
\hline Left & $4167(48.1 \%)$ & $2252(48.6 \%)$ & 6419 (48.3\%) \\
\hline Right & $4001(46.2 \%)$ & $2096(45.2 \%)$ & $6097(45.9 \%)$ \\
\hline Bilateral & $451(5.2 \%)$ & $267(5.8 \%)$ & $718(5.4 \%)$ \\
\hline Unknown & $36(0.4 \%)$ & $19(0.4 \%)$ & $55(0.4 \%)$ \\
\hline \multicolumn{4}{|l|}{ Primary_site } \\
\hline Upper-outer & $1868(21.6 \%)$ & $1016(21.9 \%)$ & $2884(21.7 \%)$ \\
\hline Upper-inner & $467(5.4 \%)$ & $284(6.1 \%)$ & $751(5.7 \%)$ \\
\hline Lower-inner & $279(3.2 \%)$ & $134(2.9 \%)$ & $413(3.1 \%)$ \\
\hline Lower-outer & $412(4.8 \%)$ & $227(4.9 \%)$ & $639(4.8 \%)$ \\
\hline Overlapping & $1601(18.5 \%)$ & $842(18.2 \%)$ & $2443(18.4 \%)$ \\
\hline Central & $529(6.1 \%)$ & $300(6.5 \%)$ & $829(6.2 \%)$ \\
\hline Breast_NOS & $3370(38.9 \%)$ & $1771(38.2 \%)$ & $5141(38.7 \%)$ \\
\hline Other & $129(1.5 \%)$ & $60(1.3 \%)$ & $189(1.4 \%)$ \\
\hline \multicolumn{4}{|l|}{ Surgery } \\
\hline Surgery not performed & $6178(71.4 \%)$ & $3671(79.2 \%)$ & $9849(74.1 \%)$ \\
\hline Surgery performed & $2351(27.2 \%)$ & $895(19.3 \%)$ & $3246(24.4 \%)$ \\
\hline Unknown & $126(1.5 \%)$ & $68(1.5 \%)$ & $194(1.5 \%)$ \\
\hline \multicolumn{4}{|l|}{ Radiation } \\
\hline Radiotherapy not performed & $113(1.3 \%)$ & $64(1.4 \%)$ & $177(1.3 \%)$ \\
\hline Radiotherapy performed & $3038(35.1 \%)$ & $1520(32.8 \%)$ & $4558(34.3 \%)$ \\
\hline None/Unknown & $5504(63.6 \%)$ & 3050 (65.8\%) & $8554(64.4 \%)$ \\
\hline \multicolumn{4}{|l|}{ Chemotherapy } \\
\hline No/Unknown & $4571(52.8 \%)$ & 2397 (51.7\%) & $6968(52.4 \%)$ \\
\hline Yes & $4084(47.2 \%)$ & 2237 (48.3\%) & $6321(47.6 \%)$ \\
\hline \multicolumn{4}{|l|}{ Histology } \\
\hline IDC & $5421(62.6 \%)$ & 2798 (60.4\%) & $8219(61.8 \%)$ \\
\hline LC & 1077 (12.4\%) & $548(11.8 \%)$ & $1625(12.2 \%)$ \\
\hline IDLC & 386 (4.5\%) & $222(4.8 \%)$ & $608(4.6 \%)$ \\
\hline IDM & $113(1.3 \%)$ & 60 (1.3\%) & $173(1.3 \%)$ \\
\hline
\end{tabular}




\begin{tabular}{|c|c|c|c|}
\hline Mucinous & $36(0.4 \%)$ & 27 (0.6\%) & 63 (0.5\%) \\
\hline Tubular & $1(0.0 \%)$ & $2(0.0 \%)$ & $3(0.0 \%)$ \\
\hline DCM & 19 (0.2\%) & $13(0.3 \%)$ & $32(0.2 \%)$ \\
\hline Other & 1602 (18.5\%) & $964(20.8 \%)$ & $2566(19.3 \%)$ \\
\hline \multicolumn{4}{|l|}{ AJCC_T } \\
\hline T1 & $959(11.1 \%)$ & $471(10.2 \%)$ & $1430(10.8 \%)$ \\
\hline T2 & $2256(26.1 \%)$ & $1266(27.3 \%)$ & $3522(26.5 \%)$ \\
\hline Т3 & $1143(13.2 \%)$ & $688(14.8 \%)$ & 1831 (13.8\%) \\
\hline T4 & 2472 (28.6\%) & $1302(28.1 \%)$ & 3774 (28.4\%) \\
\hline TX & 1637 (18.9\%) & 791 (17.1\%) & $2428(18.3 \%)$ \\
\hline T0 & $188(2.2 \%)$ & $116(2.5 \%)$ & $304(2.3 \%)$ \\
\hline \multicolumn{4}{|l|}{ AJCC_N } \\
\hline NO & 2084 (24.1\%) & 1157 (25.0\%) & 3241 (24.4\%) \\
\hline N1 & 3492 (40.3\%) & $2009(43.4 \%)$ & $5501(41.4 \%)$ \\
\hline N2 & 881 (10.2\%) & $413(8.9 \%)$ & 1294 (9.7\%) \\
\hline N3 & $1051(12.1 \%)$ & $524(11.3 \%)$ & 1575 (11.9\%) \\
\hline NX & 1147 (13.3\%) & $531(11.5 \%)$ & $1678(12.6 \%)$ \\
\hline \multicolumn{4}{|l|}{ Grade } \\
\hline Grade I & $570(6.6 \%)$ & 339 (7.3\%) & 909 (6.8\%) \\
\hline Grade II & 3003 (34.7\%) & 1575 (34.0\%) & $4578(34.4 \%)$ \\
\hline Grade III & $2741(31.7 \%)$ & 1424 (30.7\%) & $4165(31.3 \%)$ \\
\hline Grade IV & $51(0.6 \%)$ & $9(0.2 \%)$ & $60(0.5 \%)$ \\
\hline Unknown & $2290(26.5 \%)$ & 1287 (27.8\%) & 3577 (26.9\%) \\
\hline \multicolumn{4}{|l|}{ Subtype } \\
\hline HR+/HER2- & 4959 (57.3\%) & $2682(57.9 \%)$ & 7641 (57.5\%) \\
\hline HR+/HER2+ & 1135 (13.1\%) & 675 (14.6\%) & $1810(13.6 \%)$ \\
\hline HR-/HER2+ & $446(5.2 \%)$ & $258(5.6 \%)$ & 704 (5.3\%) \\
\hline HR-/HER2- & $698(8.1 \%)$ & $357(7.7 \%)$ & 1055 (7.9\%) \\
\hline Unknown & $1417(16.4 \%)$ & $662(14.3 \%)$ & $2079(15.6 \%)$ \\
\hline
\end{tabular}




\begin{tabular}{|llll|}
\hline Bone_extra & & & \\
\hline 0 & $4827(55.8 \%)$ & $2642(57.0 \%)$ & $7469(56.2 \%)$ \\
\hline 1 & $2617(30.2 \%)$ & $1351(29.2 \%)$ & $3968(29.9 \%)$ \\
\hline 3 & $913(10.5 \%)$ & $502(10.8 \%)$ & $1415(10.6 \%)$ \\
\hline Unknown & $142(1.6 \%)$ & $91(2.0 \%)$ & $233(1.8 \%)$ \\
\hline
\end{tabular}


Table 2.

Multivariate Analysis of the Training Cohort

\begin{tabular}{|c|c|c|c|}
\hline Variable & HR & $95 \% \mathrm{Cl}$ & p-value \\
\hline \multicolumn{4}{|l|}{ Age (years) } \\
\hline$<40$ & 1 & [Reference] & \\
\hline $40-49$ & 1.06 & $0.93-1.22$ & 0.361 \\
\hline $50-59$ & 1.26 & $1.11-1.43$ & $P<0.001$ \\
\hline $60-69$ & 1.4 & $1.24-1.59$ & $P<0.001$ \\
\hline $70-79$ & 1.58 & $1.39-1.8$ & $P<0.001$ \\
\hline$>=80$ & 2.34 & $2.04-2.68$ & $P<0.001$ \\
\hline \multicolumn{4}{|l|}{ Race } \\
\hline White & 1 & [Reference] & \\
\hline Black & 1.27 & $1.18-1.36$ & $P<0.001$ \\
\hline Hispanic & 0.99 & $0.91-1.08$ & 0.821 \\
\hline Asian/Pacific Islander & 0.94 & $0.84-1.05$ & 0.276 \\
\hline Other & 0.73 & $0.53-1.02$ & 0.063 \\
\hline \multicolumn{4}{|l|}{ Laterality } \\
\hline Left & 1 & [Reference] & \\
\hline Right & 0.94 & $0.9-0.99$ & 0.022 \\
\hline Bilateral & 0.8 & $0.71-0.92$ & 0.001 \\
\hline Unknown & 0.57 & $0.39-0.83$ & 0.004 \\
\hline \multicolumn{4}{|l|}{ Surgery } \\
\hline Surgery not performed & 1 & [Reference] & \\
\hline Surgery performed & 0.65 & $0.6-0.69$ & $P<0.001$ \\
\hline Unknown & 0.73 & $0.58-0.91$ & 0.006 \\
\hline \multicolumn{4}{|l|}{ Chemotherapy } \\
\hline No/Unknown & 1 & [Reference] & \\
\hline Yes & 0.7 & $0.66-0.74$ & $P<0.001$ \\
\hline \multicolumn{4}{|l|}{ AJCC T } \\
\hline T1 & 1 & [Reference] & \\
\hline
\end{tabular}




\begin{tabular}{|c|c|c|c|}
\hline $\mathrm{T} 2$ & 1.09 & $0.99-1.19$ & 0.088 \\
\hline T3 & 1.2 & $1.08-1.34$ & 0.001 \\
\hline $\mathrm{T} 4$ & 1.29 & $1.17-1.42$ & $P<0.001$ \\
\hline TX & 1.27 & $1.14-1.41$ & $P<0.001$ \\
\hline TO & 0.91 & $0.74-1.11$ & 0.353 \\
\hline \multicolumn{4}{|l|}{ AJCC N } \\
\hline NO & 1 & [Reference] & \\
\hline $\mathrm{N} 1$ & 1.01 & $0.94-1.08$ & 0.837 \\
\hline N2 & 1.05 & $0.95-1.16$ & 0.352 \\
\hline N3 & 1.13 & $1.03-1.23$ & 0.011 \\
\hline NX & 1.05 & $0.96-1.15$ & 0.259 \\
\hline \multicolumn{4}{|l|}{ Grade } \\
\hline Grade I & 1 & [Reference] & \\
\hline Grade II & 1.16 & $1.04-1.3$ & 0.009 \\
\hline Grade III & 1.57 & $1.4-1.77$ & $P<0.001$ \\
\hline Grade IV & 2.03 & $1.49-2.77$ & $P<0.001$ \\
\hline Unknown & 1.23 & $1.09-1.39$ & 0.001 \\
\hline \multicolumn{4}{|l|}{ Subtype } \\
\hline HR+/HER2- & 1 & [Reference] & \\
\hline $\mathrm{HR}+/ \mathrm{HER} 2+$ & 0.84 & $0.77-0.91$ & $P<0.001$ \\
\hline HR-/HER2+ & 1.03 & $0.91-1.17$ & 0.592 \\
\hline HR-/HER2- & 2.54 & $2.31-2.78$ & $P<0.001$ \\
\hline Unknown & 1.41 & $1.31-1.52$ & $P<0.001$ \\
\hline \multicolumn{4}{|c|}{ Extra metastatic sites } \\
\hline No & 1 & [Reference] & \\
\hline One & 1.52 & $1.43-1.61$ & $P<0.001$ \\
\hline Two & 2.29 & $2.11-2.49$ & $P<0.001$ \\
\hline Three & 2.98 & $2.48-3.57$ & $P<0.001$ \\
\hline Unknown & 1.21 & $1.01-1.46$ & 0.036 \\
\hline
\end{tabular}


Figures

Points

Age group

Race

Chemotherapy

Surgery

Laterality

Grade

Subtype

AJCC T

AJCC N

Extra metastasis except bone

Total Points

1-Year Survival Probability

3-year Survival Probability

5-year Survival Probability

$\begin{array}{lllllllllll}0 & 10 & 20 & 30 & 40 & 50 & 60 & 70 & 80 & 90 & 100\end{array}$

$40-49 \quad 60-69$
$<40=80$
Asian/Pacific Islander Hispanic
$70^{\prime}-79$

Asian/Pacific Islander Hispanic
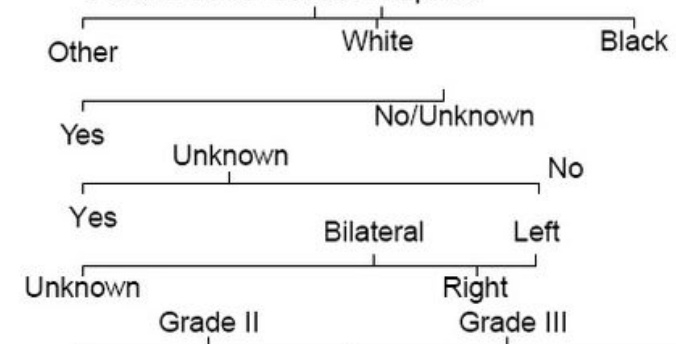

Grade I Unknown Unknown Grade IV

\begin{tabular}{|c|c|c|}
\hline $\begin{array}{c}\mathrm{HR}+/ \mathrm{HER} 2+ \\
\mathrm{T} 1\end{array}$ & $\begin{array}{c}\mathrm{HR}-/ \mathrm{HER} 2+ \\
\mathrm{T} 3\end{array}$ & HR-/HER2- \\
\hline
\end{tabular}

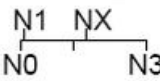

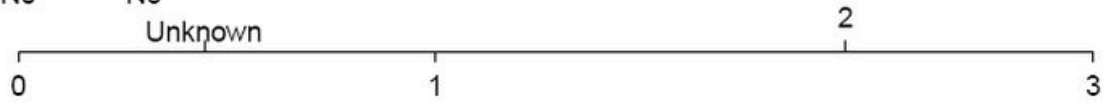

50 100 $150 \quad 200 \quad 250 \quad 300 \quad 350$ '

$\begin{array}{llllllllllll}0 & 50 & 100 & 150 & 200 & 250 & 300 & 350 & 400 & 450 & 500 & 550\end{array}$

$\begin{array}{llllllllllllllll}0.95 & 0.850 .80 & 0.70 & 0.6 & 0.5 & 0.4 & 0.3 & 0.2 & 0.1\end{array}$

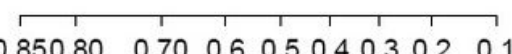

$\begin{array}{lllllllll}0.80 & 0.70 & 0.6 & 0.5 & 0.4 & 0.3 & 0.2 & 0.1\end{array}$

\section{Figure 1}

Nomograms for predicting 1-year, 3-year, and 5-year overall survival (OS) of breast cancer patients with bone metastasis. 
A

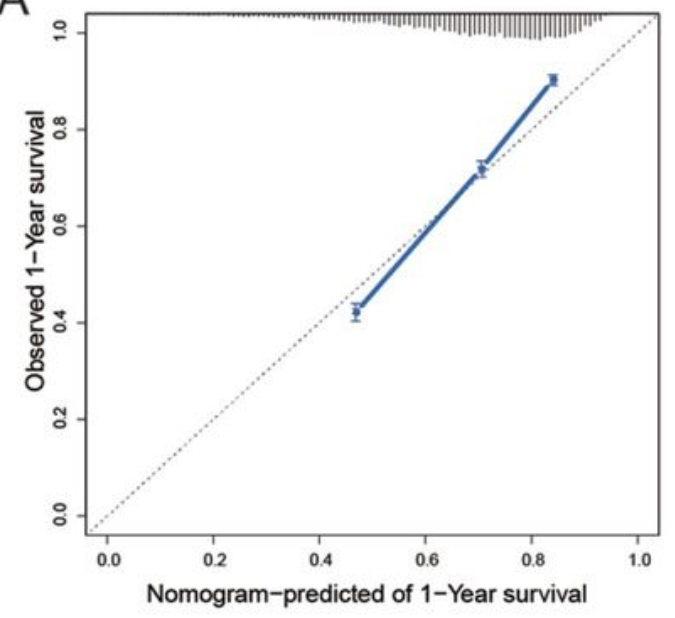

C

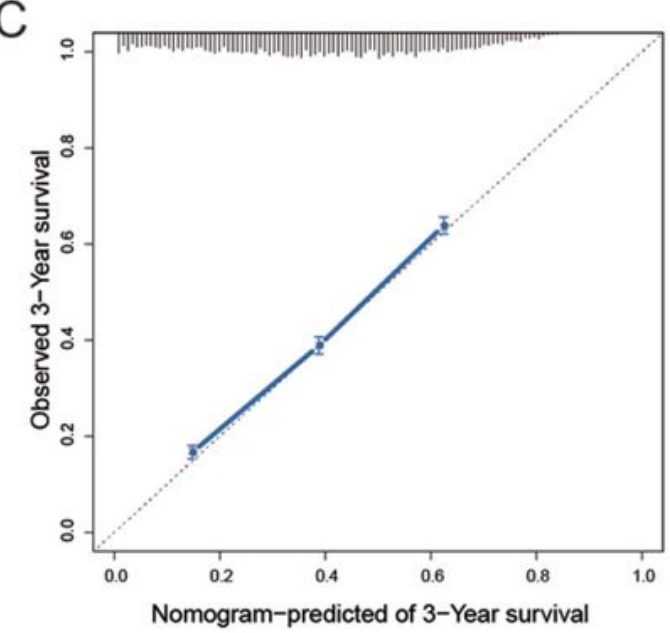

$\mathrm{E}$

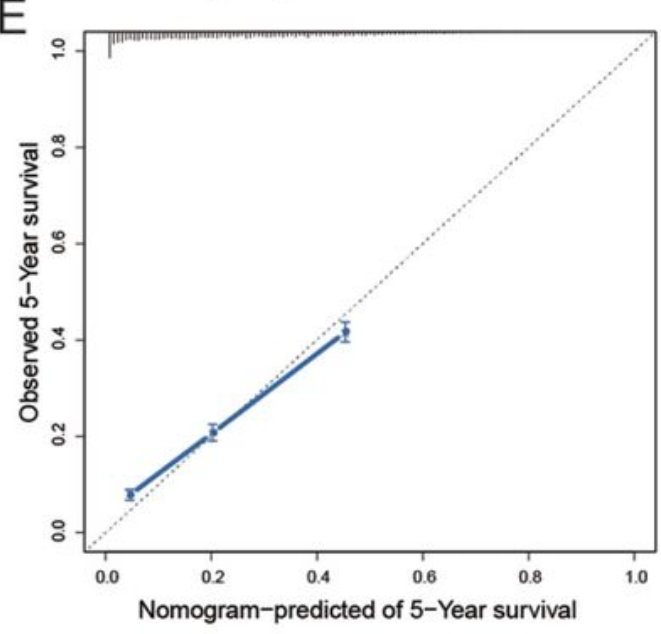

$\mathrm{B}$

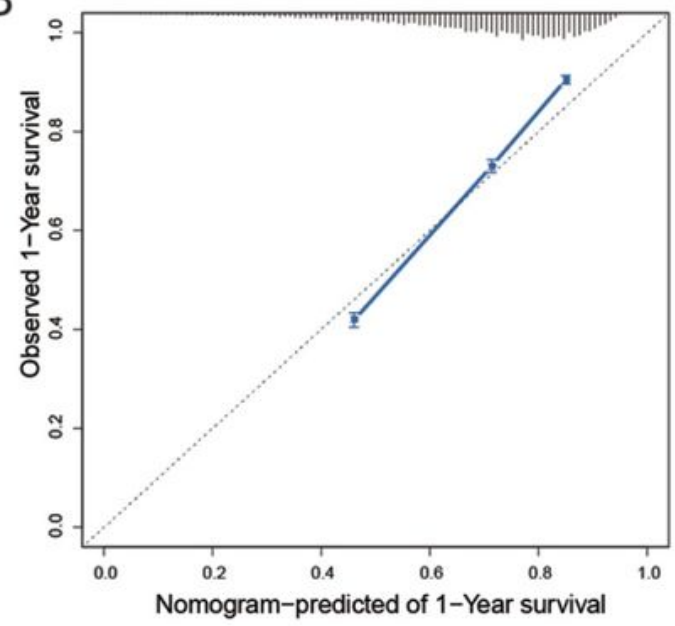

D

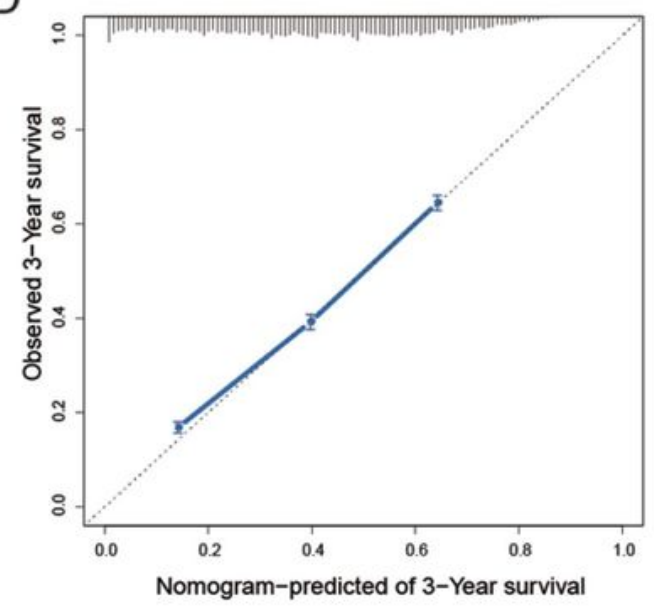

$\mathrm{F}$

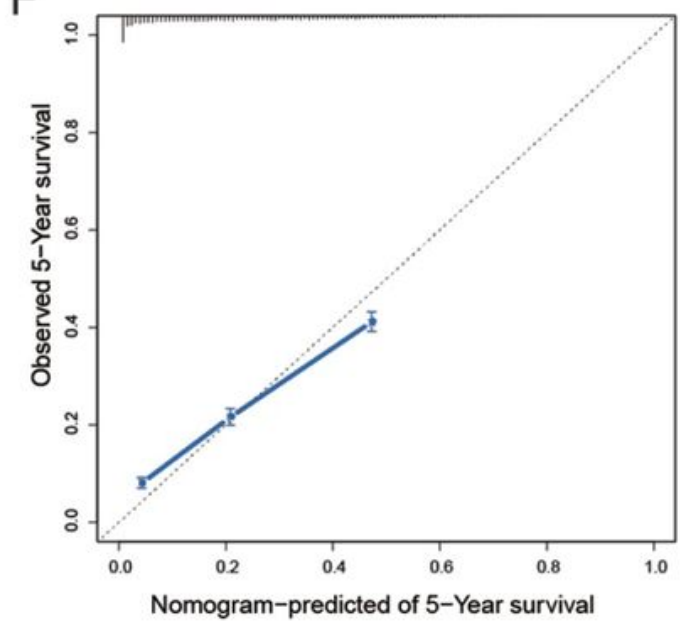

\section{Figure 2}

The calibration curve for predicting patient survival at (A) 1 year, (C)3 years, $(E)$ and 5 years in the training cohort and at (B, D, F) 1 year, 3 years, 5 years in the validation cohort. Nomogram-predicted probability of overall survival is plotted on the $x$-axis; actual overall survival is plotted on the $y$-axis.

\section{Supplementary Files}


This is a list of supplementary files associated with this preprint. Click to download.

- TableS1.xlsx 